\title{
Puberty Is Delayed in Male Mice With Dextran Sodium Sulfate Colitis Out of Proportion to Changes in Food Intake, Body Weight, and Serum Levels of Leptin
}

\author{
MARK D. DEBOER AND YONGLI LI \\ Department of Pediatrics, University of Virginia, Charlottesville, Virginia 22908
}

\begin{abstract}
In boys, inflammatory bowel disease often results in delayed puberty associated with decreased bone mineral density and decreased linear growth. Our goal was to investigate whether pubertal timing and levels of leptin differed between prepubertal male mice with colitis and food-restricted (FR) mice maintained at a similar weight. We induced colitis in 32-d-old male mice using dextran sodium sulfate (DSS), resulting in $10 \mathrm{~d}$ of worsening colitis. We followed up these mice for separation of the prepuce from the glans penis as a marker of pubertal progression. Compared with freefeeding control mice, DSS and FR mice had significantly lower weight on d 7-10 of treatment. DSS mice had later puberty than control and FR mice. DSS mice also had smaller testes, lower FSH levels, increased systemic cytokines, and increased colonic inflammation by histology. Leptin levels were similar between DSS and FR mice, whereas both had decreases in leptin compared with controls. We conclude that DSS colitis causes delayed puberty in sexually immature male mice beyond what is seen among FR mice of similar weight, food intake, and leptin levels. These experiments provide support for the hypothesis that pubertal delay in colitis is influenced by factors beyond poor weight gain alone. (Pediatr Res 69: 34-39, 2011)
\end{abstract}

$\mathrm{I}_{\mathrm{e}}^{\mathrm{n}}$ nflammatory bowel disease (IBD), including Crohn's disease and ulcerative colitis, is associated with a delay in puberty in boys $(1,2)$ that can lead to decreased bone mineral accrual and a loss of a pubertal growth spurt $(1,3,4)$. The mechanism of this delay in puberty is thought to be at least in part because of the malnutrition seen in colitis (5). In other states of malnutrition, depleted fat stores are associated with low levels of leptin, a hormone produced by adipocytes in proportion to the amount of fat within the cells (6). Leptin is known to be necessary for pubertal progression, as evidenced by a lack of pubertal progression in rodent (7) and human $(8,9)$ males with leptin deficiency. Thus, one potential mechanism for pubertal delay in males with colitis could be decreased fat stores leading to lower leptin levels (10).

Although a previous model of colitis in prepubertal male rats [using trinitrobenzene sulfonic acid (TNBS)] revealed a trend toward later puberty among rats with colitis versus a pair-fed group of similar weight, it is not known whether levels of leptin differed between prepubertal animals with

Received May 5, 2010; accepted September 9, 2010.

Correspondence: Mark D. DeBoer, M.D., Department of Pediatrics, University of Virginia, P.O. Box 800386, Charlottesville, VA 22908; e-mail: deboer@virginia.edu Supported by National Institutes of Health Grant HD060739-01 [M.D.D.] and by the University of Virginia Silvio O. Conte Digestive Health Research Center and the Pediatric Endocrine Society grants. colitis versus food-restricted (FR) controls (11). We have previously demonstrated a difference in the timing of puberty in prepubertal female mice with colitis compared with a FR group (12). However, influences on the timing of puberty are known to differ between males and females in relationship to body weight, as illustrated by a tendency for overweight females to have earlier puberty $(13,14)$ compared with a tendency for overweight males to have later puberty (15). Similar gender-based differences may exist in the context of low body weight as well. Thus, the effects of colitis and leptin on pubertal timing in male mice remain unclear.

Given these gaps in knowledge, our goal was to determine 1) whether male mice with colitis indeed have later puberty than FR mice of a similar weight and 2) whether male mice with colitis have lower levels of leptin as a potential explanation for their delayed puberty. To determine these issues, we induced colitis in prepubertal male mice via administration of $3 \%$ dextran sodium sulfate (DSS) (16) in the drinking water and followed up the mice for the separation of the prepuce from the glans penis $(11,17,18)$. These experiments continue a line of research investigating whether there are processes besides undernutrition that contribute to delayed puberty in the setting of colitis.

\section{MATERIALS AND METHODS}

These experiments were approved by the Animal Care and Use Committee at the University of Virginia. C57BL6 mice were purchased from Jackson Laboratories and were housed in standard wire-top cages and fed with phytoestrogenfree chow. Two females and one male of childbearing age were housed together for breeding. Pups from litters of 6-10 mice were included in these experiments. Pups were weaned at the age of $19 \mathrm{~d}$ by being removed from mother and placed in a cage with moistened chow for $3 \mathrm{~d}$ before starting dry food exclusively.

Starting at day of life (DOL) 32, male mice were evenly divided based on starting weight into three groups: control, DSS colitis, and FR. DSS colitis mice had 3\% DSS $\left(M_{\mathrm{r}}, 36 ; 100-45,500\right.$; TdB Consultancy, Uppsala, Sweden) placed in the water supply from DOL 32-39 before DSS treatment was discontinued, and animals were allowed to experience continued colitis from DOL 39 to 42. Control mice and FR mice received deionized water alone throughout the course of the experiment.

Both control and DSS mice were given phytoestrogen-free food ad libitum throughout the experiment. FR mice were given only enough food to maintain their weight at the same level as the DSS-treated group. This food administration for the FR group was done on an empirical basis each day for each mouse in the FR group, whose daily weight as a percent of baseline was compared with the DSS mean.

Abbreviations: DOL, day of life; DSS, dextran sodium sulfate; FR, food restricted; GnRH, gonadotropin-releasing hormone; IBD, inflammatory bowel disease; KC, cytokine-induced neutrophil chemoattractant; TNBS, trinitrobenzene sulfonic acid 
A
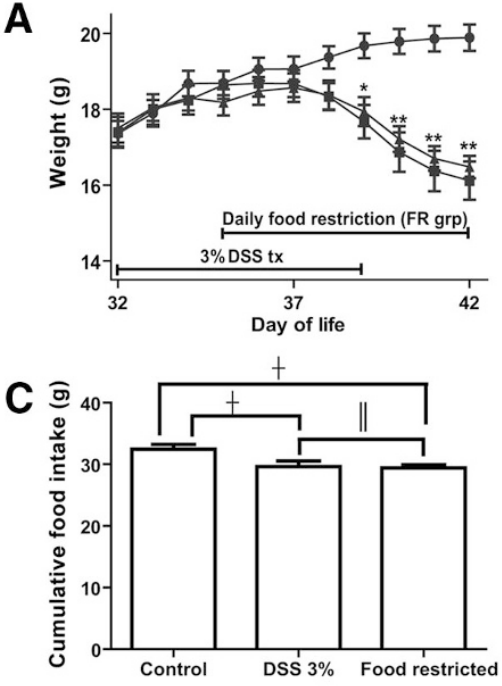

B

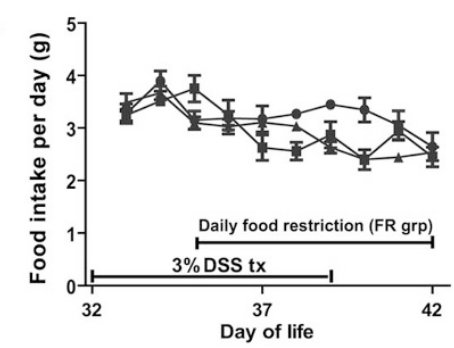

D

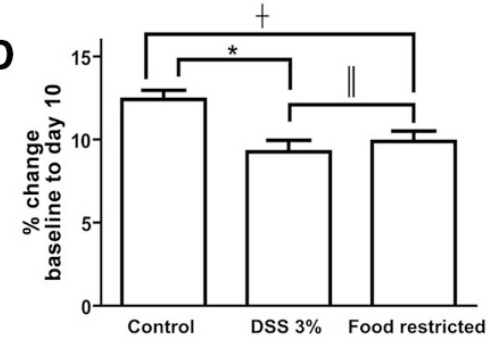

Figure 1. Weight, length, and food intake after colitis induction and food restriction. (A) Weight curve and $(B)$ food intake over the course of the experiment, DOL 32-42 for control (circles, $n=$ 16 ), DSS (squares, $n=17$ ), and FR mice (triangles, $n=16$ ). (C) Ten-day cumulative food intake during experiment. $(D)$ Linear growth determined as follows: (length at DOL 42)/(length at DOL 32). Significance $v s$ control: $\dagger p<0.05, * p<0.01$, $* * p<0.001 ; \|$ not significant $(p>0.05)$.

Observation for complete separation of prepuce and glans penis was performed by daily inspection using surgical loupes and recorded as the day on which the glans penis was completely retracted from the prepuce $(11,17,18)$. Although every effort was made to blind the assessment of prepuce-glans separation, it is acknowledged that this was difficult given clear differences in the mice themselves related to bloody diarrhea in the DSS-treated mice and weight loss among both DSS- and FR groups. Levels of LH and FSH before and after gonadotropin-releasing hormone $(\mathrm{GnRH})$ stimulation were determined in separate groups of control, DSS, and FR mice treated as described earlier on DOL 42. GnRH stimulation was performed using $100 \mathrm{mg} / \mathrm{kg}$ of $\mathrm{GnRH}$ (Bachem Americas, Inc, Torrence, CA) injected s.c., with blood drawn $15 \mathrm{~min}$ after injection. In addition, separate groups of control, DSS, and FR mice were used to measure intermediate serum levels of leptin and cytokines. Serum samples were drawn from these mice on DOL 36 and DOL 39, and the mice were killed on DOL 42.

At time of killing, animals were injected with standard mouse cocktail followed by cardiac puncture. Serum was stored at $-80^{\circ}$ until time of testing. Colons were removed and fixed in Bouin's reagent before embedding in paraffin, sectioning, and staining. Slides from individual animals were read by a blinded pathologist (James Mize, M.D., Anatomic and Clinical Pathology, Fairfax, VA) and scored for acute Inflammatory Index and Chronic Inflammatory Index (19).

Serum measurements. Serum cytokines were tested in the University of Virginia Digestive Health Research Center core using a Beadlyte multiplex system (Upstate Cell Signaling Solutions, Temecula, CA). Because of limitations in the amount of serum volume, not all serum hormone measurements were able to be performed for all animals but were performed on randomly selected mice in each treatment group. Serum hormone measurements were tested in the University of Virginia Center for Research in Reproduction. Testosterone was measured via RIA (DPC Products, Corp., Los Angeles, CA). LH was measured in serum by a modified supersensitive two-site sandwich immunoassay, and FSH was determined by RIA, both measurements performed as described previously (12). Corticosterone was performed using a Corticosterone Coat-a-Count kit (Siemens Healthcare Diagnostics, Los Angeles, CA).

Serum leptin measurement was performed using ELISA (Chrystal Chem, Inc., Downers Grove, IL; sensitivity $0.05 \mathrm{ng} / \mathrm{mL}$; inter-/intraassay variability, $3.0-$ $4.6 \% / 1.1-1.8 \%$, respectively). Leptin measurements were performed in singlicate for all animals in all groups.

Statistics. Statistics were calculated using Prizm spreadsheet software and SAS. Comparisons of mean values between the three groups were performed using ANOVA with Bonferroni posttest comparison of all groups. KaplanMeier curves of timing of prepuce-glans separation were analyzed via logrank test (20).

\section{RESULTS}

Weight gain, linear growth, and food intake. Prepubertal male mice receiving 3\% DSS between DOL 32 and 39 exhibited a decrease in weight relative to controls starting at DOL 39 and continuing until mice were killed at DOL 42 (Fig. 1A). This decreased weight/weight loss relative to
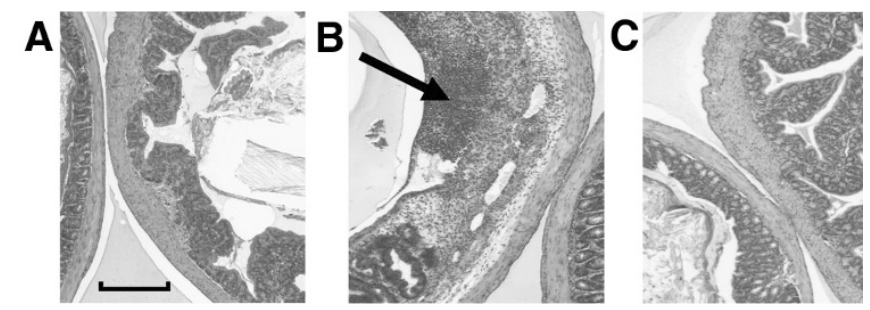

Figure 2. Colonic histology. Representative histological sections of colons from mice from the control $(A)$, DSS $(B)$, and FR $(C)$ groups; arrow indicates local inflammation and ulceration in the DSS-treated mouse $(\times 10$ magnification and bar equals $0.25 \mathrm{~mm}$ ).

controls was matched in the FR group. Food intake during the 10-d experiment was overall similar among the DSS and FR groups, and both groups had a decreased food intake relative to the control group (Fig. $1 B$ and $C$ ).

Linear growth was affected for the DSS group relative to control (Fig. 1D). Linear growth for the FR group was intermediate between the control and DSS groups, with no significant difference between the FR group and DSS or control mice.

Colon histology. The DSS group exhibited an increase in colonic inflammation with Active Inflammatory Index Score and Chronic Inflammatory Index Score of $2.65 \pm 0.47$ and $2.60 \pm 0.47$, respectively; both control and FR groups had scores of $0 \pm 0(p<0.001$; Fig. $2 A-C)$.

Cytokines. Starting at $7 \mathrm{~d}$ of treatment (DOL 39), the DSS group had significant increases in the systemic inflammatory cytokines IL-6 compared with other groups (Fig. 3A) and cytokine-induced neutrophil chemoattractant (KC; Fig. 3B). There were no significant differences at any time point for TNF- $\alpha$ (Fig. 3C). Data shown are fold increase above control. Serum concentration of cytokines in control mice were as follows: IL-6, $21.4 \pm 6.3 \mathrm{pg} / \mathrm{mL}, n=16$; KC, $38.5 \pm 8.3$ $\mathrm{pg} / \mathrm{mL}, n=16$; and TNF- $\alpha, 11.7 \pm 2.1 \mathrm{pg} / \mathrm{mL}, n=16$. There were no differences in levels of IL- $1 \beta$ or the anti-inflammatory cytokine IL-10 (data not shown). 

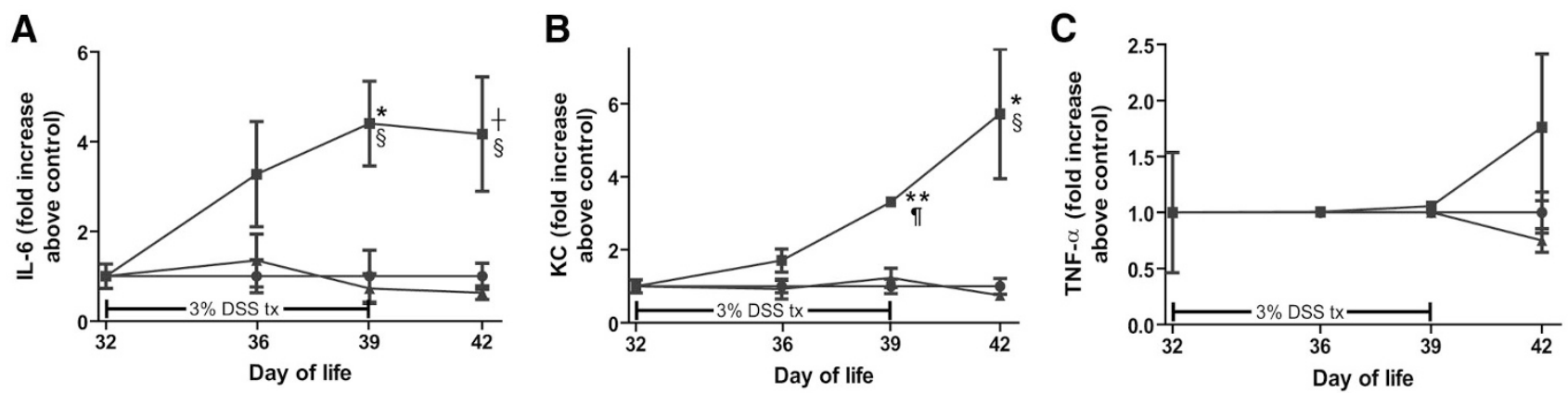

Figure 3. Systemic inflammation. Serum measurement of cytokines at baseline (DOL 32), midpoints (DOL 36 and 39), and the end of experiment (DOL 42), expressed as fold change above control for control (circles), DSS (squares), and FR mice (triangles). Midpoint values were performed in separate groups of animals under the same protocol. See text for absolute serum values. (A) IL-6, (B) KC, and (C) TNF- $\alpha$. Significance $v s$ control: $\dagger p<0.05$, $* p<0.01$, $* * p<$ 0.001 ; significance $v s$ FR: $\$ p<0.01, \mathbb{I} p<0.001$.
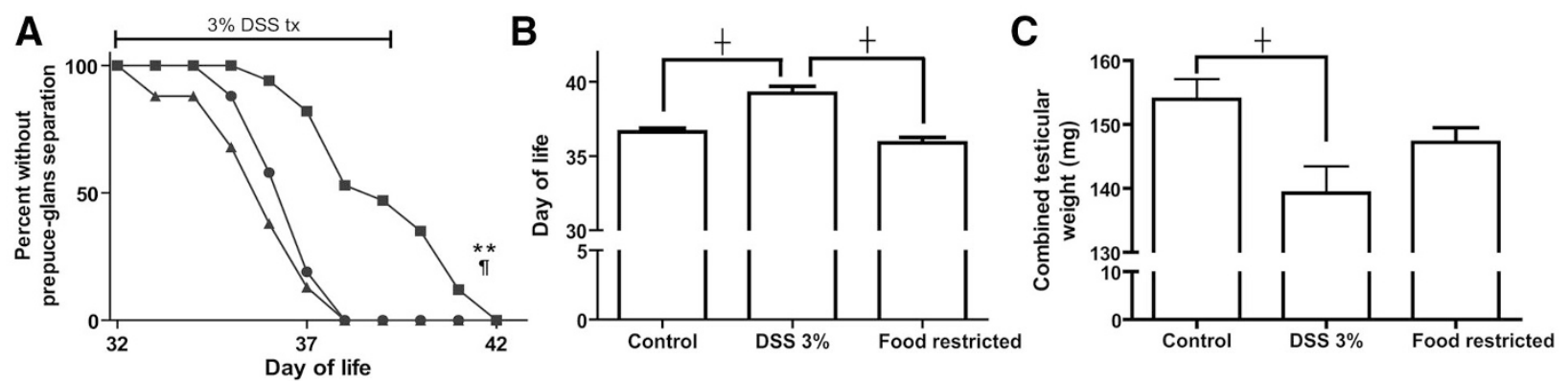

Figure 4. Timing of prepuce-glans separation. (A) Kaplan-Meier curve showing the proportion of mice without prepuce-glans separation over time as assessed by daily examination for control (circles), DSS (squares), and FR mice (triangles). (B) Mean age of prepuce-glans separation. (C) Testicular weight on DOL 42. $\dagger p<0.05 ; * * p<0.001$ DSS $v s$ control and $\mathbb{I} p<0.001$ vs FR as determined by log-rank test.
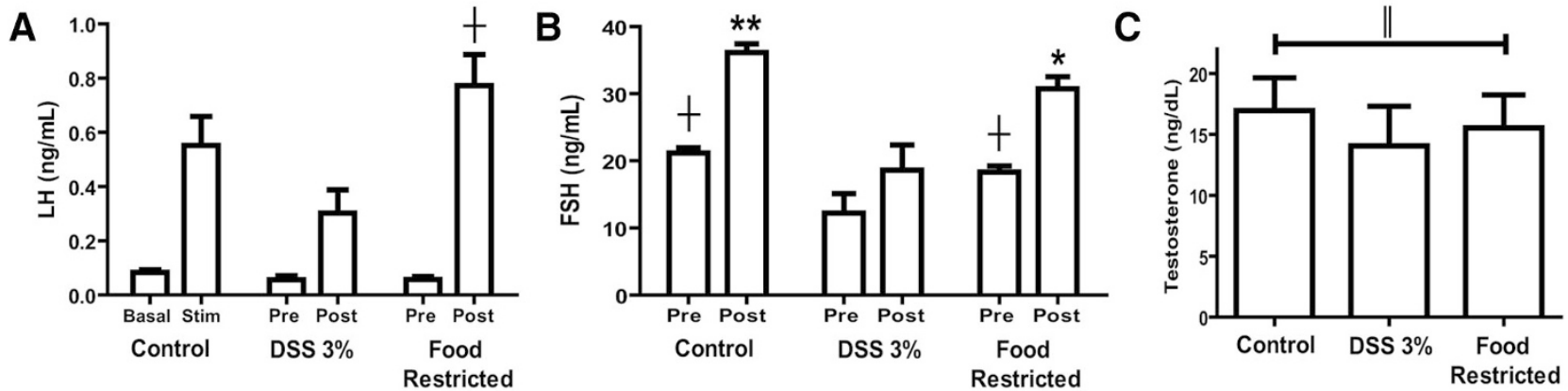

Figure 5. Reproductive hormone levels. (A) Levels of LH at baseline ("basal": control, $n=9$; DSS, $n=8$; and FR, $n=11$ ) and after GnRH stimulation ("stim": control, $n=10$; DSS, $n=6$; and FR, $n=10$ ). (B) Levels of FSH: basal (control, $n=11$; DSS, $n=7$; and FR, $n=8$ ) and after GnRH stimulation (control, $n=10$; DSS, $n=6$; and FR, $n=10$ ), all as measured on DOL 42; GnRH-stimulated values are on a separate group of animals under the same protocol. ( $C$ ) Testosterone on DOL 42 (control, $n=14$; DSS, $n=8$; and FR, $n=10$ ). The $p$ values $v s$ corresponding DSS group: $\dagger p<0.05 ; * p<0.01, * * p<0.001 ; \|$ not significant $(p>0.05)$.

Prepuce-glans separation and testicular weights. The DSS group exhibited a delay in prepuce-glans separation relative to both the control group and the FR group, as analyzed both by Kaplan-Meier curve plotting percent animals without prepuceglans separation (Fig. 4A) and by mean age of prepuce-glans separation (Fig. 4B).

The DSS group had a significant decrease in testicular weight compared with the control group (Fig. 4C). There were no significant differences in testicular weight between control and FR mice or between DSS and FR mice. Histological comparison of testicular sections from each treatment group failed to reveal differences in signs of inflammation or sertoli cell appearance between the DSS, control, and FR groups (data not shown).

Hormone levels. There were no significant differences in baseline levels of LH between the DSS group and either the control or FR groups (Fig. 5A). After GnHR stimulation, each group had higher levels of LH than at baseline $(p<0.01)$. FR mice had higher GnRH-stimulated levels of LH than DSS mice. Levels of FSH were lower in the DSS colitis group compared with the control and FR group both at baseline and after GnRH stimulation (Fig. 5B). There were no significant 
differences in testosterone between the groups, with the majority of values being at the low end of the measurable range, near the limit of detection 8.5 (Fig. $5 C$ ).

Leptin levels remained stable from baseline until DOL 36 in all groups but were decreased from baseline starting at DOL 39 for both the DSS and FR groups (Fig. 5A). Leptin levels for DSS and FR animals were lower than control levels from DOL 40 to 42 . There were no differences in leptin levels between the DSS and FR groups at any time point. On DOL 42, there was no significant difference in corticosterone levels between any of the groups (Fig. 5B).

\section{DISCUSSION}

Puberty marks a time of rapid physical and psychological change separating childhood from adulthood. Puberty is often seen as a surrogate for general medical health because multiple chronic illnesses-including IBD_frequently result in delayed puberty (21). This delay is associated with decreased bone density and may result in a loss of final height potential (1), underscoring the clinical importance of determining the mechanisms behind delayed puberty in IBD. One proposed mechanism for this delay is that IBD causes decreased food intake and poor weight gain, leading to low levels of leptin-a hormone necessary for the onset of puberty (1,8-10). As a means of investigating this issue, we have used a model of DSS colitis in prepubertal male mice to demonstrate that these mice have a delay in puberty relative to mice that were FR to maintain the same weight as the DSS group. Although it was theoretically possible that male mice with colitis would have lower levels of leptin than FR mice as an explanation for the further delay in puberty, we instead found that DSS and FR mice had similar levels of leptin throughout the course of the experiment, suggesting that changes in reproductive hormone regulation among male mice with colitis was related to factors besides low leptin alone-although low leptin levels may potentiate the effects of additional factors. These findings are similar to our previous report of delayed puberty among female mice with DSS colitis versus FR mice (12). In contrast to gender differences in the effect of body weight on pubertal timing in the context of overweight (13-15), these findings suggest a similar pattern of delayed puberty in male and female mice beyond what would be expected by leptin levels alone. In addition, this study is unique in that no previous investigators have demonstrated a delay in puberty among male animals with colitis relative to a group with similar weights.

In this manner, our results contrast with the findings of Azooz et al. (11). These researchers used TNBS to induce colitis in sexually immature male rats and compared these with a pair-fed group. They reported a nonsignificant trend toward later puberty TNBS group with pubertal delay in 57\% of rats with colitis versus $28 \%$ of the pair-fed group. The major difference between our study and that of Azooz et al. is the model of colitis used. In the study by Azooz et al., rats with TNBS colitis had an earlier onset of low body weight relative to controls, with a significant weight difference starting only $3 \mathrm{~d}$ after TNBS administration and continuing for the remainder of the experiments ( $14 \mathrm{~d}$ total of colitis). In their experiments, the pair-fed rats had a daily weight curve that overlapped almost completely with the TNBS group, indicating that poor weight gain in that model was due almost entirely to a decrease in food intake among the TNBS rats. The rapid separation in weight between the control rats and both the TNBS and pair-fed rats seemed to delay puberty similarly among these two groups.

By contrast, we found a significant difference in pubertal timing between DSS-treated mice and FR mice. The reason for this difference in pubertal timing may relate to the weight curve that we observed compared with the weight curve in the study of Azooz et al. Similar to IBD seen in children, our administration of DSS resulted in a more insidious onset of poor weight gain. Although the previous investigators noted a separation in weights at d 3 of colitis, we did not note a significant weight difference until d 7 of the experiment (Fig. $1 A$ ). In the interval between $\mathrm{d} 1$ of colitis and $\mathrm{d} 7$-during which the control, DSS, and FR groups all had similar body weight (Fig. 1A) and similar levels of leptin (Fig. 6A)-100\% of control and FR mice exhibited separation of the prepuce from the glans penis (Fig. $4 A$ and $B$ ). The main factors separating the DSS-treated mice from the control and FR mice during this time range were the worsening colonic and systemic inflammation (Fig. $3 A$ and $B$ ). It may be that early changes in inflammation contributed to the delay of puberty in these experiments, as has been suggested previously $(12,22)$ and has been suggested as a cause of decreased fertility in men with IBD (23).

There were differences noted between specific hormone responses between male mice with colitis and female mice with colitis (12). Female mice exhibited an initial rise in leptin
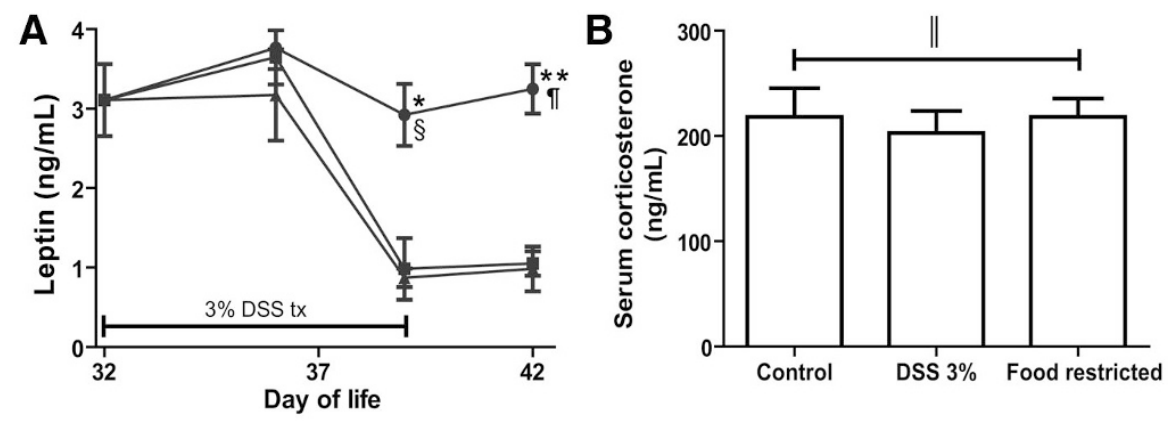

Figure 6. Serum levels of leptin and corticosterone. (A) Serum levels of leptin at the baseline, midpoints, and end of experiment. Midpoint values were performed in separate groups of animals under the same protocol, with equal FR and DSS animal weights. (B) Serum levels of corticosterone at the end of experiment (DOL 42). Significance $v s$ DSS: $* p<0.01, * * p<$ 0.001 ; significance $v s$ FR: $\$ p<0.01, \mathbb{T} p<$ $0.001 ; \|$ not significant $(p>0.05)$. 
levels by d 4 of colitis, whereas males merely maintained initial leptin levels. This may be due to differences in fat mass acquisition in that male control and DSS mice had gained an average of $7.4 \%$ of initial weight by $d 4$, whereas female control and DSS mice had gained $21 \%$ by this time. Female mice in the FR group had higher levels of corticosterone, compared with males who showed no difference in corticosterone between groups, of uncertain cause.

The male mice with colitis in our experiment demonstrated an increase in systemic inflammation relative to both of the other two groups, including increased levels of the inflammatory cytokines IL- 6 and KC. IL- 6 has been shown to be a major factor in the systemic disease associated with colitis in that mice and humans that receive infusions of antibodies to IL-6 show improvements in colitis-related factors such as colitis symptoms $(24,25)$, growth (26), and bone metabolism (27). Similarly, production of KC in the colon has been demonstrated during DSS colitis in adult mice (28), and administration of anti-KC antibodies during TNBS colitis resulted in decreased colonic inflammation and improved survival (29). We did not observe a significant difference in TNF- $\alpha$ levels during colitis. However, antibodies to TNF- $\alpha$ are widely used in the treatment of colitis and have been shown to be associated with improvements in colitis-related sequelae such as growth (30). In consideration of the timing of these increases in systemic cytokines, IL-6 levels had already exhibited a trend toward increased levels among the DSS group by DOL 36 (Fig. $2 A, p=0.059$ ), during the timing of delayed puberty.

The case for systemic inflammation causing changes in gonadotropin regulation-and thus changes in the onset puberty-is strengthened by multiple previous experiments that demonstrated a suppressive effect of experimental inflammation on release of gonadotropins. Administration of inflammatory cytokines via intracerebroventricular injection $(31,32)$ and peripheral injection of lipopolysaccharide (33) have been shown to decrease systemic levels of LH and FSH, which are in turn responsible for stimulating the production of testosterone in the testes. Because puberty proceeds by the same process (gonadotropin-driven testosterone production), the suppression of gonadotropins by systemic inflammation provides a plausible explanation for the delayed pubertal timing of mice with DSS colitis relative to controls. Although we did not note a difference in basal levels of LH or testosterone between mice with DSS colitis and control animals, it must be noted that 1) LH is normally secreted in bursts, and basal levels may not be indicative of the overall area under the curve for LH over the course of the experiment and 2) testosterone values in our study were all near the limit of detection for the assay used $(8.5 \mathrm{ng} / \mathrm{dL})$ including in control animals, making it difficult to show a true difference between groups. We did note lower levels of LH after GnRH stimulation among DSS-treated mice. In addition, our validated test of testosterone exposure [i.e. the separation of the prepuce from the glans penis $(11,17,18)]$ suggests that testosterone levels were lower over the course of the experiment in DSS-treated mice.

In addition to decreases in gonadotropin-stimulated production of testosterone, an alternate explanation for a delay in pubertal progression is through direct effects of colitis on testicular production of testosterone. As mentioned, we did not note a difference in testosterone levels between our mice, with all of our mice having testosterone levels near the limit of detection. In addition, if the mice with DSS colitis had a decrease in testosterone production because of the direct effects of colitis on testicular function, we would expect to see a secondary increase in LH levels as the animal attempted to further increase production. This is seen in mice who have received orchiectomies at a similar age (34). The absence of such a secondary increase in LH provides evidence that the delay in puberty was not because of a direct effect on gonadal function.

We noted a decrease in testicular size among mice with DSS colitis, which may have been because of their delay in puberty. It may also have been because of a decrease in FSH. Normally, FSH stimulates the sertoli cells in the testis, constituting a large proportion of testicular volume (35). Our mice with DSS colitis had both lower FSH and smaller testes than control mice and did not exhibit differences in testicular morphology on histological examination. Overall, the lower FSH levels and smaller testes are consistent both with their delayed sexual maturation and with a decrease in gonadotropin production related to their colitis.

The most common explanation for delayed puberty in colitis is the decrease in food consumed or absorbed (1). Although we did not directly assess the degree of absorption of food, we found similar total food intake among mice with DSS colitis and those who had been FR. Unlike the study by Azooz et al., we did not systematically feed our FR mice the same amount of food as the colitis mice. Instead, we empirically chose an amount of food that allowed their weight curve to match the curve of the mice with colitis. Interestingly, it turned out that the total food consumed did not differ overall between the two groups. In this manner, they were similar to the study by Azooz et al., potentially suggesting that the major inhibition on weight gain in DSS colitis-as in TNBS colitis in rats-is anorexia and not a decrease in food absorption. Nevertheless, it must be noted that neither we nor Azooz et al. measured basal metabolic rates between groups. Changes in basal metabolic rates may also be an important factor in that, e.g. a decrease in food absorption could be offset by a similar decrease in energy expenditure. Overall, we found that despite similar rates of food intake, mice with DSS colitis had a later timing of puberty than the FR group, again suggesting a cause other than decreased food intake.

In conclusion, we noted a delayed timing of puberty in male mice with DSS colitis relative to FR mice with similar weight and leptin levels, suggesting that low levels of leptin were not the primary cause of pubertal delay. Further studies are needed to better define the exact means by which colitis produces this pubertal delay and whether therapeutic interventions to prevent this delay result in improved outcomes related to bone density and final height.

Acknowledgments. We thank Dr. Steven Cohn, M.D., $\mathrm{Ph} . \mathrm{D}$. , and his laboratory for their assistance in the induction and evaluation of colitis in these experiments. We also thank 
Dr. Matthew Gurka, Ph.D., for assistance with statistical evaluation.

\section{REFERENCES}

1. Ballinger AB, Savage MO, Sanderson IR 2003 Delayed puberty associated with inflammatory bowel disease. Pediatr Res 53:205-210

2. Brain CE, Savage MO 1994 Growth and puberty in chronic inflammatory bowel disease. Baillieres Clin Gastroenterol 8:83-100

3. Paganelli M, Albanese C, Borrelli O, Civitelli F, Canitano N, Viola F, Passariello R, Cucchiara S 2007 Inflammation is the main determinant of low bone mineral density in pediatric inflammatory bowel disease. Inflamm Bowel Dis 13:416-423

4. DeBoer MD, Barnes BH 2009 The importance of treatment regimens and pubertal status for growth in IBD. J Pediatr 154:936-937

5. Bousvaros A, Sylvester F, Kugathasan S, Szigethy E, Fiocchi C, Colletti R, Otley A, Amre D, Ferry G, Czinn SJ, Splawski JB, Oliva-Hemker M, Hyams JS, Faubion WA, Kirschner BS, Dubinsky MC 2006 Challenges in pediatric inflammatory bowel disease. Inflamm Bowel Dis 12:885-913

6. Ahima RS, Prabakaran D, Mantzoros C, Qu D, Lowell B, Maratos-Flier E, Flier JS 1996 Role of leptin in the neuroendocrine response to fasting. Nature 382:250-252

7. Smith JT, Acohido BV, Clifton DK, Steiner RA 2006 KiSS-1 neurones are direct targets for leptin in the ob/ob mouse. J Neuroendocrinol 18:298-303

8. Farooqi IS 2002 Leptin and the onset of puberty: insights from rodent and human genetics. Semin Reprod Med 20:139-144

9. Farooqi IS, Jebb SA, Langmack G, Lawrence E, Cheetham $\mathrm{CH}$, Prentice AM, Hughes IA, McCamish MA, O'Rahilly S 1999 Effects of recombinant leptin therapy in a child with congenital leptin deficiency. N Engl J Med 341:879-884

10. Kaminski BA, Palmert MR 2008 Genetic control of pubertal timing. Curr Opin Pediatr 20:458-464

11. Azooz OG, Farthing MJ, Savage MO, Ballinger AB 2001 Delayed puberty and response to testosterone in a rat model of colitis. Am J Physiol Regul Integr Comp Physiol 281:R1483-R1491

12. DeBoer MD, Li Y, Cohn S 2010 Colitis causes delay in puberty in female mice out of proportion to changes in leptin and corticosterone. J Gastroenterol 45:277-284

13. Adair LS, Gordon-Larsen P 2001 Maturational timing and overweight prevalence in US adolescent girls. Am J Public Health 91:642-644

14. Kaplowitz PB 2008 Link between body fat and the timing of puberty. Pediatrics 121:S208-S217

15. Lee JM, Kaciroti N, Appugliese D, Corwyn RF, Bradley RH, Lumeng JC 2010 Body mass index and timing of pubertal initiation in boys. Arch Pediatr Adolesc Med 164:139-144

16. Wirtz S, Neufert C, Weigmann B, Neurath MF 2007 Chemically induced mouse models of intestinal inflammation. Nat Protoc 2:541-546

17. Kolho KL, Huhtaniemi I 1989 Neonatal treatment of male rats with a gonadotropinreleasing hormone antagonist results in altered function of the pituitary-testicular axis in adult age. Biol Reprod 41:1084-1090
18. Korenbrot CC, Huhtaniemi IT, Weiner RI 1977 Preputial separation as an external sign of pubertal development in the male rat. Biol Reprod 17:298-303

19. Marini M, Bamias G, Rivera-Nieves J, Moskaluk CA, Hoang SB, Ross WG, Pizarro TT, Cominelli F 2003 TNF-alpha neutralization ameliorates the severity of murine Crohn's-like ileitis by abrogation of intestinal epithelial cell apoptosis. Proc Natl Acad Sci U S A 100:8366-8371

20. Bland JM, Altman DG 2004 The logrank test. BMJ 328:1073

21. Simon D 2002 Puberty in chronically diseased patients. Horm Res 57:53-56

22. Wong SC, Macrae VE, McGrogan P, Ahmed SF 2006 The role of pro-inflammatory cytokines in inflammatory bowel disease growth retardation. J Pediatr Gastroenterol Nutr 43:144-155

23. Feagins LA, Kane SV 2009 Sexual and reproductive issues for men with inflammatory bowel disease. Am J Gastroenterol 104:768-773

24. Ito H, Takazoe M, Fukuda Y, Hibi T, Kusugami K, Andoh A, Matsumoto T, Yamamura T, Azuma J, Nishimoto N, Yoshizaki K, Shimoyama T, Kishimoto T 2004 A pilot randomized trial of a human anti-interleukin-6 receptor monoclonal antibody in active Crohn's disease. Gastroenterology 126:989-996

25. Yamamoto M, Yoshizaki K, Kishimoto T, Ito H 2000 IL-6 is required for the development of Th1 cell-mediated murine colitis. J Immunol 164:4878-4882

26. Sawczenko A, Azooz O, Paraszczuk J, Idestrom M, Croft NM, Savage MO, Ballinger AB, Sanderson IR 2005 Intestinal inflammation-induced growth retardation acts through IL- 6 in rats and depends on the -174 IL- 6 G/C polymorphism in children. Proc Natl Acad Sci U S A 102:13260-13265

27. Uno JK, Kolek OI, Hines ER, Xu H, Timmermann BN, Kiela PR, Ghishan FK 2006 The role of tumor necrosis factor alpha in down-regulation of osteoblast Phex gene expression in experimental murine colitis. Gastroenterology 131:497-509

28. Froicu M, Cantorna MT 2007 Vitamin D and the vitamin D receptor are critical for control of the innate immune response to colonic injury. BMC Immunol 8:5

29. Bento AF, Leite DF, Claudino RF, Hara DB, Leal PC, Calixto JB 2008 The selective nonpeptide CXCR2 antagonist SB225002 ameliorates acute experimental colitis in mice. J Leukoc Biol 84:1213-1221

30. Walters TD, Gilman AR, Griffiths AM 2007 Linear growth improves during infliximab therapy in children with chronically active severe Crohn's disease. Inflamm Bowel Dis 13:424-430

31. Kalra PS, Fuentes M, Sahu A, Kalra SP 1990 Endogenous opioid peptides mediate the interleukin-1-induced inhibition of the release of luteinizing hormone ( $\mathrm{LH}$ )releasing hormone and LH. Endocrinology 127:2381-2386

32. Rivier C, Vale W 1990 Cytokines act within the brain to inhibit luteinizing hormone secretion and ovulation in the rat. Endocrinology 127:849-856

33. Nappi RE, Rivest S 1997 Effect of immune and metabolic challenges on the luteinizing hormone-releasing hormone neuronal system in cycling female rats: an evaluation at the transcriptional level. Endocrinology 138:1374-1384

34. Huang BM, Leu SF, Yang HY, Norman RL 2001 Testosterone effects on luteinizing hormone and follicle-stimulating hormone responses to gonadotropin-releasing hormone in the mouse. J Androl 22:507-513

35. Sairam MR, Krishnamurthy H 2001 The role of follicle-stimulating hormone in spermatogenesis: lessons from knockout animal models. Arch Med Res 32:601-608 